\title{
Asymptotic Behavior of Bounded Solutions TO A First ORDER GRADIENT-LIKE System
}

\author{
Minh-Phuong TRAN $N^{1}$, Thanh-Nhan NGUYEN $N^{2, *}$ \\ ${ }^{1}$ Applied Analysis Research Group, Faculty of Mathematics and Statistics, Ton Duc Thang \\ University, Ho Chi Minh City, Vietnam \\ ${ }^{2}$ Department of Mathematics, Ho Chi Minh City University of Education, Ho Chi Minh City, \\ Vietnam \\ *Corresponding Author: Thanh-Nhan NGUYEN (Email: nhannt@hcmue.edu.vn) \\ (Received: 26-Dec-2018; accepted: 25-Feb-2019; published: 31-Mar-2019) \\ DOI: http://dx.doi.org/10.25073/jaec.201931.227
}

\begin{abstract}
In this paper, we prove the long time behavior of bounded solutions to a first order gradient-like system with low damping and perturbation terms. Our convergence results are obtained under some hypotheses of KurdykaLojasiewicz inequality and the angle and comparability condition.
\end{abstract}

\section{Keywords}

Asymptotic Behavior, Gradient-Like System, Convergence to Equilibrium.

\section{Introduction}

The main goal of this paper is to obtain the asymptotic behavior of bounded solutions to the gradient-like system as follows

$$
u^{\prime}(t)+\gamma(t) u(t)+G(u(t))=f(t), t \in[0, \infty),
$$

where the unknown $u(t) \in \mathbb{R}^{n}$, the damping term $\gamma \in L^{1}\left(\mathbb{R}^{+}, \mathbb{R}^{+}\right)$, the perturbation term $f \in L^{1}\left(\mathbb{R}^{+}, \mathbb{R}^{n}\right)$ and $G \in C\left(\mathbb{R}^{n}, \mathbb{R}^{n}\right)$ is a tangent vector field on $\mathbb{R}^{n}$. Roughly speaking, we study in this paper the effect of adding a low damping term $\gamma(t) u(t)$ and a perturbation term $f(t)$ to the equation

$$
u^{\prime}(t)+G(u(t))=0, t \in[0, \infty),
$$

on the long time behavior of the trajectories $u$.

This type of problem have been studied in many recent papers with different assumptions of $G$. The typical situation of (2) is the case of gradient system when $G=\nabla F$. This gradient system was studied by many authors such as [1], [6], [14], [15], [18] or [21]. In the classical result, they proved that the bounded solution converges to an equilibrium as $t$ goes to infinity if the function $F$ is real analytic in [18]. More later, R. Chill et al. [8] established an general result which guarantees that the convergence result also holds for the gradient-like system (2). This convergence result was proved under the hypotheses of the Lojasiewicz inequality of $F$ and the angle condition of $G$ and $\nabla F$. In [19] and [20], the authors extended the result by Kurdyka-Lojasiewicz inequality. Moreover, the convergence rates was obtained if $F$ satisfies Lojasiewicz inequality and $G, \nabla F$ satisfy angle and comparability condition.

Recently, R. Chill and M. Jendoubi [7] or Huang and Takac [17] considered the equation in the non homogeneous case. They showed that any bounded solution of the gradient system

$$
\dot{u}+\nabla F(u)=f(t), \quad t \geq 0
$$


converges to a critical point of $F$ at infinity under the following condition

$$
\sup _{t \in \mathbb{R}^{+}} t^{1+\mu} \int_{0}^{\infty}\|f(s)\|^{2} \mathrm{~d} s<\infty,
$$

for some positive constant $\mu$. The forcing term $f(t)$ quickly decays to zero as $t$ goes to infinity in this case. In a previous work [22], we proved the convergence result of (3) under a low $L^{1}$-condition of the perturbation term. Moreover, the rate of convergence was even obtained under a Lojasiewicz inequality of the Lyapunov function $F$. The convergence results have been generalized to some second order systems, such as [2], [4], [5], [12] or [13] are references therein. Moreover, M. Ghisi et. al. have estimated the decay rates for solutions of semi linear dissipative equations in [9] and [10].

Motivated by these works, we establish in this paper the convergence results for the first order non homogeneous gradient-like system (1) with the effect of a low damping and forcing terms. More precisely, we consider the equation (1) with $\gamma$ and $f$ satisfy the following condition

$$
\int_{0}^{\infty}\left(\int_{t}^{\infty} \gamma^{2}(s) \mathrm{d} s\right)^{1 / 2} \mathrm{~d} t<\infty
$$

and

$$
\int_{0}^{\infty}\left(\int_{t}^{\infty}\|f(s)\|^{2} \mathrm{~d} s\right)^{1 / 2} \mathrm{~d} t<\infty
$$

In addition, we also assume some key hypotheses such as the angle and comparability condition of $G$ and $\nabla F$ and the Kurdyka-Lojasiewicz inequality of $F$ as in many other articles. The nice feature of angle and comparability condition of $G$ and $\nabla F$ is that $G$ is coincident with the gradient of $F$ with respect to a Riemannian metric $g$. We refer the reader to the article of Barta et. al. [3] for the detail. Under these assumptions, we prove that the bounded solution $u$ to equation (1) converges to a critical point $\varphi \in \omega[u]$ at infinity and $\dot{u} \in L^{1}\left(\mathbb{R}^{+}\right)$.

The paper is organized as follows. In the next section, we present some assumptions and definitions that we use through the whole of the paper. We also recall the existence of a Riemannian metric $g$ such that $G=\nabla_{g} F$ in this section.
In the last section, we establish the asymptotic behavior of bounded solutions to gradient-like system (1). Our results are divided into three theorems for the convenience of the reader.

\section{Preliminaries}

In this section, we give the key assumption of angle and comparability condition to obtain the convergence result of gradient-like system. We also recall some definitions about the Lyapunov function, the Kurdyka-Lojasiewicz inequality and the gradient of a function with respect to a Riemannian metric.

We consider a continuous tangent vector field $G \in C\left(\mathbb{R}^{n}, \mathbb{R}^{n}\right)$ respects to a Lyapunov function $F \in C^{1}\left(\mathbb{R}^{n}, \mathbb{R}\right)$. In this paper, we always assume the angle and comparability condition of $\nabla F$ and $G$, i.e, there exists a positive constant $a>0$ such that for any $u \in \mathbb{R}^{n}$ there holds

$$
\langle G(u), \nabla F(u)\rangle \geq a\left(\|G(u)\|^{2}+\|\nabla F(u)\|^{2}\right),
$$

where $\langle\cdot, \cdot\rangle$ and $\|\cdot\|$ denote the inner product and Euclidean norm in $\mathbb{R}^{n}$ respectively.

Definition 1. F is called a Lyapunov function for equation (1) if

$$
\langle G(u), \nabla F(u)\rangle \geq 0, \forall u \in \mathbb{R}^{n} .
$$

Moreover, we say that $F$ is a strict Lyapunov function if $\nabla F(u)=0$ implies $G(u)=0$.

We remark that $F$ is a strict Lyapunov function if the angle and comparability condition (5) holds.

Definition 2. We say that the function $F$ satisfies a Kurdyka-Lojasiewicz inequality at $\eta$ if there exist $\delta>0$ and a non decreasing function $\Theta \in C\left(\mathbb{R}^{+}, \mathbb{R}^{+}\right)$such that

$$
\Theta(0)=0, \Theta^{-1} \in L_{l o c}^{1}\left(\mathbb{R}^{+}\right)
$$

and

$$
\Theta(|F(u)-F(\eta)|) \leq\|\nabla F(u)\|, \forall u \in B_{\delta}(\eta),
$$

where $B_{\delta}(\eta)$ denotes the ball centered at $\eta$ and radius $\delta$ in $\mathbb{R}^{n}$. 
This definition is related to the Lojasiewicz Theorem in [18] below.

Theorem 1. If $F$ is real analytic in a neighborhood of $\eta$ then $F$ satisfies the KurdykaLojasiewicz inequality (7) at $\eta$.

We denote by $\nabla_{g(u)} F(u)$ the gradient of $F$ with respect to a Riemannian metric $g$ on $\mathbb{R}^{n}$ at $u$, i.e., for any $v \in \mathbb{R}^{n}$, we have

$$
\langle\nabla F(u), v\rangle=\left\langle\nabla_{g(u)} F(u), v\right\rangle_{g(u)} .
$$

For simplicity, we write $\nabla_{g} F(u)$ instead of $\nabla_{g(u)} F(u)$ and $\langle\cdot, \cdot\rangle_{g}$ instead of $\langle\cdot, \cdot\rangle_{g(u)}$. We denote by $\|\cdot\|_{g}$ the induced norm. In [3], the authors showed that there exists a Riemannian metric $g$ which is equivalent to the Euclidean metric such that $G=\nabla_{g} F$. We recall this result in the following theorem.

Theorem 2. Assume the angle and comparability condition (5) of $\nabla F$ and $G$ holds. Then there exists a Riemannian metric $g$ on

$$
V:=\left\{u \in \mathbb{R}^{n}: G(u) \neq 0\right\}
$$

such that $G=\nabla_{g} F$.

Moreover, there exist positive constants $\alpha, \beta$ such that

$$
\alpha\|v\| \leq\|v\|_{g(u)} \leq \beta\|v\|,
$$

for any $v \in \mathbb{R}^{n}, u \in V$ and $\|\cdot\|$ denotes the Euclidean metric on $\mathbb{R}^{n}$.

Definition 3. For any trajectory $u$ belongs to $C\left(\mathbb{R}^{+}, \mathbb{R}^{n}\right)$, the $\omega$-limit set of $u$ is defined by

$$
\omega[u]=\left\{\varphi \in \mathbb{R}^{n}: \exists\left(t_{m}\right) \uparrow \infty, u\left(t_{m}\right) \rightarrow \varphi\right\} .
$$

\section{Main results}

In this section, we prove the convergence of bounded solutions to equilibrium of the gradient-like system (1). The main idea of our work is based on Theorem 2. Applying Theorem 2, the gradient-like system (1) can be seen as a form of gradient system.

Theorem 3. Let $u$ be a bounded solution of (1) and $f, \gamma \in L^{2}\left(\mathbb{R}^{+}\right)$. Assume that the angle and comparability condition (5) of $G$ and $\nabla F$ holds. Then $\dot{u} \in L^{2}\left(\mathbb{R}^{+}\right)$and $G(u) \in L^{2}\left(\mathbb{R}^{+}\right)$.
Proof. Let us consider the energy function defined by

$\Phi(t)=F(u(t))+\frac{1}{2} \int_{t}^{\infty}\|\gamma(s) u(s)-f(s)\|_{g(u(s))}^{2} \mathrm{~d} s$.

The function $\Phi$ is well defined. Indeed, applying Theorem 2 for $G$ and $\nabla F$ under the angle and comparability condition (5), we have $G=\nabla_{g} F$ and the inequality (9) holds. Combining with $f, \gamma \in L^{2}\left(\mathbb{R}^{+}\right)$, we then obtain that

$$
\begin{aligned}
& \int_{t}^{\infty}\|\gamma(s) u(s)-f(s)\|_{g(u(s))}^{2} \mathrm{~d} s \\
& \leq \beta^{2} \int_{t}^{\infty}\|\gamma(s) u(s)-f(s)\|^{2} \mathrm{~d} s \\
& \leq 2 \beta^{2} \sup _{t \in[0, \infty)}\|u(t)\| \int_{t}^{\infty}|\gamma(s)|^{2} \mathrm{~d} s \\
& \quad+2 \beta^{2} \int_{t}^{\infty}\|f(s)\|^{2} \mathrm{~d} s \\
& \leq C\left(\|\gamma\|_{L^{2}\left(\mathbb{R}^{+}\right)}^{2}+\|f\|_{L^{2}\left(\mathbb{R}^{+}\right)}^{2}\right) \\
&<\infty
\end{aligned}
$$

where $C=2 \beta^{2} \max \left\{1, \sup _{t \in[0, \infty)}\|u(t)\|\right\}$.

For every $t \in \mathbb{R}^{+}$, we have

$$
\begin{aligned}
\frac{\mathrm{d}}{\mathrm{d} t} \Phi(t)= & \langle\nabla F(u(t)), \dot{u}\rangle \\
& -\frac{1}{2}\|\gamma(t) u(t)-f(t)\|_{g}^{2} \\
= & \left\langle\nabla_{g} F(u(t)), \dot{u}\right\rangle_{g} \\
& -\frac{1}{2}\|\gamma(t) u(t)-f(t)\|_{g}^{2} .
\end{aligned}
$$

By the angle and comparability (5), equation (1) and Theorem 2, we can estimate the derivative of the energy function $\Phi$ as follows

$$
\begin{aligned}
\frac{\mathrm{d}}{\mathrm{d} t} \Phi(t) & =\langle G(u(t)), \dot{u}\rangle_{g}-\frac{1}{2}\|\dot{u}+G(u(t))\|_{g}^{2} \\
& =-\frac{1}{2}\left(\|\dot{u}\|_{g}^{2}+\|G(u(t))\|_{g}^{2}\right) \\
& \leq-\frac{a}{2}\left(\|\dot{u}\|^{2}+\|G(u(t))\|^{2}\right) \\
& \leq 0 .
\end{aligned}
$$


It deduces that $\Phi$ is non increasing and therefore

$$
\begin{aligned}
\int_{0}^{\infty}\|\dot{u}(s)\|^{2} \mathrm{~d} s & \leq-\frac{2}{a} \int_{0}^{\infty} \frac{\mathrm{d}}{\mathrm{d} t} \Phi(s) \mathrm{d} s \\
& \leq \frac{2}{a}(\Phi(0)-\Phi(\infty)) \\
& <\infty
\end{aligned}
$$

which implies that $\dot{u} \in L^{2}\left(\mathbb{R}^{+}\right)$.

Similarly, we also obtain that $G(u) \in L^{2}\left(\mathbb{R}^{+}\right)$. The proof is complete.

Theorem 4. Under the hypotheses of Theorem 3, then $\nabla_{g} F(\varphi)=0$ for any $\varphi \in \omega[u]$.

Proof. Since $u$ is a bounded solution of equation (1), so the $\omega$-limit set $\omega[u]$ is non empty.

Let $\varphi \in \omega[u]$, there exists a sequence $\left(t_{m}\right)_{m \in \mathbb{N}}$ such that $t_{m} \uparrow \infty$ and $u\left(t_{m}\right)$ tends to $\varphi$ as $m$ goes to infinity.

By Theorem 3, we have

$$
\int_{t}^{t+1}\|\dot{u}(s)\|^{2} \mathrm{~d} s \rightarrow 0 \text { as } t \rightarrow \infty
$$

Thanks to Cauchy-Schwartz inequality, we obtain

$$
\begin{aligned}
\sup _{s \in[0,1]}\left\|\int_{t_{m}}^{t_{m}+s} \dot{u}(r) \mathrm{d} r\right\| \\
\leq \sup _{s \in[0,1]} \int_{t_{m}}^{t_{m}+s}\|\dot{u}(r)\| \mathrm{d} r \\
\quad=\int_{t_{m}}^{t_{m}+s}\|\dot{u}(r)\| \mathrm{d} r \\
\leq\left(\int_{t_{m}}^{t_{m}+s}\|\dot{u}(r)\|^{2} \mathrm{~d} r\right)^{1 / 2},
\end{aligned}
$$

which goes to 0 as $m$ goes to infinity. This means

$$
u\left(t_{m}+s\right)=u\left(t_{m}\right)+\int_{t_{m}}^{t_{m}+s} \dot{u}(r) \mathrm{d} r \rightrightarrows \varphi
$$

where $\rightrightarrows$ denotes the uniformly convergence. Using the continuity of $\nabla F$, we obtain that $\nabla F\left(u\left(t_{m}+s\right)\right)$ uniformly converges to $\nabla F(\varphi)$ in $[0,1]$.
Moreover, for any $v \in \mathbb{R}^{n}$, we have

$$
\begin{aligned}
& \left|\int_{t_{m}}^{t_{m}+1}\langle\dot{u}(s), v\rangle_{g(u(s))} \mathrm{d} s\right| \\
& \leq \int_{t_{m}}^{t_{m}+1}\left|\langle\dot{u}(s), v\rangle_{g(u(s))}\right| \mathrm{d} s \\
& \quad \leq \beta^{2} \int_{t_{m}}^{t_{m}+1}\|\dot{u}(s)\|\|v\| \mathrm{d} s \\
& \leq \beta^{2}\|v\|\left(\int_{t_{m}}^{t_{m}+1}\|\dot{u}(s)\|^{2} \mathrm{~d} s\right)^{1 / 2},
\end{aligned}
$$

which tends to 0 as $m$ goes to infinity. Similarly, we also get

$$
\begin{aligned}
& \left|\int_{t_{m}}^{t_{m}+1}\langle\gamma(s) u(s), v\rangle_{g(u(s))} \mathrm{d} s\right| \\
& \quad \leq \int_{t_{m}}^{t_{m}+1}\left|\langle\gamma(s) u(s), v\rangle_{g(u(s))}\right| \mathrm{d} s \\
& \quad \leq \beta^{2} \int_{t_{m}}^{t_{m}+1}\|\gamma(s) u(s)\|\|v\| \mathrm{d} s \\
& \leq M \beta^{2}\|v\|\left(\int_{t_{m}}^{t_{m}+1}\|\gamma(s)\|^{2} \mathrm{~d} s\right)^{1 / 2},
\end{aligned}
$$

and

$$
\begin{aligned}
& \left|\int_{t_{m}}^{t_{m}+1}\langle f(s), v\rangle_{g(u(s))} \mathrm{d} s\right| \\
& \quad \leq \int_{t_{m}}^{t_{m}+1}\left|\langle f(s), v\rangle_{g(u(s))}\right| \mathrm{d} s \\
& \quad \leq \beta^{2} \int_{t_{m}}^{t_{m}+1}\|f(s)\|\|v\| \mathrm{d} s \\
& \quad \leq M \beta^{2}\|v\|\left(\int_{t_{m}}^{t_{m}+1}\|f(s)\|^{2} \mathrm{~d} s\right)^{1 / 2}
\end{aligned}
$$

where $M$ is an upper bound of the bounded solution $u$. So we can conclude that the right hand sides vanish as $m$ goes to infinity. 
Combining these estimations and equation (1), it follows that

$$
\begin{aligned}
\int_{t_{m}}^{t_{m}+1}\langle & G(u(s)), v\rangle_{g(u(s))} \mathrm{d} s \\
= & -\int_{t_{m}}^{t_{m}+1}\langle\dot{u}(s), v\rangle_{g(u(s))} \mathrm{d} s \\
& -\int_{t_{m}}^{t_{m}+1}\langle\gamma(s) u(s), v\rangle_{g(u(s))} \mathrm{d} s \\
& +\int_{t_{m}}^{t_{m}+1}\langle f(s), v\rangle_{g(u(s))} \mathrm{d} s,
\end{aligned}
$$

which tends to 0 as $m$ goes to infinity.

Finally, to finish the proof, we can present the inner product of $\nabla F(\varphi)$ and $v$ for any $v \in \mathbb{R}^{n}$ as follows

$$
\begin{aligned}
& \langle\nabla F(\varphi), v\rangle=\int_{0}^{1}\langle\nabla F(\varphi), v\rangle \mathrm{d} s \\
& =\lim _{m \rightarrow \infty} \int_{0}^{1}\left\langle\nabla F\left(u\left(t_{m}+s\right)\right), v\right\rangle \mathrm{d} s \\
& =\lim _{m \rightarrow \infty} \int_{t_{m}}^{t_{m}+1}\langle\nabla F(u(s)), v\rangle \mathrm{d} s \\
& =\lim _{m \rightarrow \infty} \int_{t_{m}}^{t_{m}+1}\left\langle\nabla_{g(u)} F(u(s)), v\right\rangle_{g(u(s))} \mathrm{d} s \\
& =\lim _{m \rightarrow \infty} \int_{t_{m}}^{t_{m}+1}\langle G(u(s)), v\rangle_{g(u(s))} \mathrm{d} s \\
& =0 .
\end{aligned}
$$

This equality shows that $\nabla_{g} F(\varphi)=0$.

Theorem 5. Assume that

i) the angle and comparability condition (5) of $G$ and $\nabla F$ holds;

ii) F satisfies the Kurdyka-Lojasiewicz inequality (7) and the function $\Theta$ in (6)-(7) satisfies

$$
\Theta(x+y) \leq k \Theta(x)+|y|^{1 / 2}, \forall x, y \in \mathbb{R},
$$

for some positive constant $k$;

iii) $\gamma \in L^{1}\left(\mathbb{R}^{+}\right) \cap L^{2}\left(\mathbb{R}^{+}\right)$such that

$$
\int_{0}^{\infty}\left(\int_{t}^{\infty} \gamma^{2}(s) d s\right)^{1 / 2} d t<\infty
$$

iv) $f \in L^{1}\left(\mathbb{R}^{+}, \mathbb{R}^{n}\right) \cap L^{2}\left(\mathbb{R}^{+}, \mathbb{R}^{n}\right)$ such that

$$
\int_{0}^{\infty}\left(\int_{t}^{\infty}\|f(s)\|^{2} d s\right)^{1 / 2} d t<\infty
$$

If $u$ is a bounded solution to equation (1) then $\dot{u} \in L^{1}\left(\mathbb{R}^{+}\right)$and $u(t)$ converges to an equilibrium point $\varphi \in \omega[u]$ at infinity.

Proof. We consider again the energy function $\Phi$ defined by (10). It is similar to the proof of Theorem 3, we also get the following estimation

$$
-\frac{\mathrm{d}}{\mathrm{d} t} \Phi(t)=\frac{1}{2}\left(\|\dot{u}\|_{g}^{2}+\|G(u(t))\|_{g}^{2}\right),
$$

for every $t \in \mathbb{R}^{+}$. It implies that the function $\Phi$ is non increasing. Moreover, since $u$ is a bounded solution, the $\omega$-limit set $\omega[u]$ is non empty. Let $\varphi \in \omega[u]$, we have $\Phi(t)$ converges to $F(\varphi)$ at infinity. We remark that by subtracting $F(\varphi)$ if needed we may assume $F(\varphi)=0$. It implies that the energy function $\Phi(t)$ is non negative for every $t \in[0, \infty)$.

It is easily to see that if $\Phi(T)=0$ for some $T \geq 0$ then $u$ is constant for all $t \geq T$. There remain nothing to prove in this case. Hence, we now assume that $\Phi(t)>0$ for every $t \in[0, \infty)$.

Since the solution $u$ is bounded, so there exists a positive constant $M$ such that

$$
\|u(t)\| \leq M, \forall t \in[0, \infty) .
$$

By assumption $i i$ ), there exist $\delta>0$ and the function $\Theta$ satisfying (6) such that for all $u \in$ $B_{\delta}(\varphi)$, there holds

$$
\Theta(|F(u)|) \leq\|\nabla F(u)\| .
$$

We now consider a function $I$ defined by

$$
I(x)=\int_{0}^{x} \frac{1}{\Theta(s)} \mathrm{d} s, \quad x \in[0, \infty)
$$

For any $\varepsilon \in(0, \delta)$, by the hypotheses (12) and (13), there exists $t^{*}$ such that

$$
\begin{gathered}
\left\|u\left(t^{*}\right)-\varphi\right\|+\frac{1}{a \alpha^{2}} I\left(\Phi\left(t^{*}\right)\right)<\varepsilon / 4, \\
\frac{a \beta M}{k} \int_{t^{*}}^{\infty}\left(\int_{s}^{\infty}|\gamma(r)|^{2} \mathrm{~d} r\right)^{1 / 2} \mathrm{~d} s<\varepsilon / 4,
\end{gathered}
$$




$$
\begin{aligned}
& \frac{a \beta}{k} \int_{t^{*}}^{\infty}\left(\int_{s}^{\infty}\|f(r)\|^{2} \mathrm{~d} r\right)^{1 / 2} \mathrm{~d} s<\varepsilon / 4, \\
& M \int_{t^{*}}^{\infty}|\gamma(s)| \mathrm{d} s+\int_{t^{*}}^{\infty}\|f(s)\| \mathrm{d} s<\varepsilon / 4 .
\end{aligned}
$$

Let us define

$$
T=\inf \left\{t \geq t^{*}:\|u(t)-\varphi\| \geq \varepsilon\right\} .
$$

For every $t \in\left[t^{*}, T\right)$, one has

$$
\frac{\mathrm{d}}{\mathrm{d} t} I(\Phi(t))=\Phi^{\prime}(t) \Theta^{-1}(\Phi(t)) .
$$

Using the definition of energy function $\Phi$ in (10) and the hypothesis (11), we obtain

$$
\begin{aligned}
& \Theta(\Phi(t)) \leq k \Theta(F(u(t))) \\
& +\frac{1}{\sqrt{2}}\left(\int_{t}^{\infty}\|\gamma(s) u(s)-f(s)\|_{g(u(s))}^{2} \mathrm{~d} s\right)^{1 / 2} .
\end{aligned}
$$

According to Kurdyka-Lojasiewicz (7) and the equivalence between Riemannian metric $g$ and Euclidean metric in (9), we have

$$
\begin{aligned}
& \Theta(\Phi(t)) \leq k\|\nabla F(u(t))\| \\
& \quad+\frac{\beta}{\sqrt{2}}\left(\int_{t}^{\infty}\|\gamma(s) u(s)-f(s)\|^{2} \mathrm{~d} s\right)^{1 / 2} .
\end{aligned}
$$

Applying the angle and comparability condition (5), we get that

$$
\Theta(\Phi(t)) \leq k a^{-1}\|G(u(t))\|+A
$$

where

$$
A=\frac{\beta}{\sqrt{2}}\left(\int_{t}^{\infty}\|\gamma(s) u(s)-f(s)\|^{2} \mathrm{~d} s\right)^{1 / 2} .
$$

Combining (16) with (15), (14) and (9), it deduces that

$$
\begin{aligned}
-\frac{\mathrm{d}}{\mathrm{d} t} I(\Phi(t)) & \geq \frac{\alpha^{2} k\|\dot{u}(t)\|\|G(u(t))\|}{k a^{-1}\|G(u(t))\|+A} \\
& =a \alpha^{2}\|\dot{u}(t)\|-\frac{a \alpha^{2} A\|\dot{u}(t)\|}{k a^{-1}\|G(u(t))\|+A} .
\end{aligned}
$$

It yields that

$$
\begin{aligned}
\|\dot{u}(t)\| \leq-\frac{1}{a \alpha^{2}} & \frac{\mathrm{d}}{\mathrm{d} t} I(\Phi(t)) \\
& +\frac{A\|\dot{u}(t)\|}{k a^{-1}\|G(u(t))\|+A} .
\end{aligned}
$$

By equation (1), we obtain

$$
\|\dot{u}(t)\| \leq \| G(u(t)\|+\| \gamma(t) u(t)-f(t) \| .
$$

This gives

$$
\begin{aligned}
\frac{A\|\dot{u}(t)\|}{k a^{-1}\|G(u(t))\|+A} \leq & \frac{a}{k} A \\
& +\|\gamma(t) u(t)-f(t)\| .
\end{aligned}
$$

In the other hand, one has

$$
\|\gamma(t) u(t)-f(t)\| \leq M|\gamma(t)|+\|f(t)\|,
$$

and

$$
\begin{aligned}
A \leq \beta M\left(\int_{t}^{\infty}|\gamma(s)|^{2} \mathrm{~d} s\right)^{1 / 2} & \\
& \quad+\beta\left(\int_{t}^{\infty}\|f(s)\|^{2} \mathrm{~d} s\right)^{1 / 2} .
\end{aligned}
$$

Integrating (17) on $\left[t^{*}, t\right)$ for any $t \in\left[t^{*}, T\right)$ we get that

$$
\begin{aligned}
\int_{t^{*}}^{t}\|\dot{u}(s)\| \mathrm{d} s & \leq \frac{1}{a \alpha^{2}} I\left(\Phi\left(t^{*}\right)\right) \\
& +\frac{a \beta M}{k} \int_{t^{*}}^{t}\left(\int_{s}^{\infty}|\gamma(r)|^{2} \mathrm{~d} r\right)^{1 / 2} \mathrm{~d} s \\
& +\frac{a \beta}{k} \int_{t^{*}}^{t}\left(\int_{s}^{\infty}\|f(r)\|^{2} \mathrm{~d} r\right)^{1 / 2} \mathrm{~d} s \\
& +M \int_{t^{*}}^{t}|\gamma(s)| \mathrm{d} s+\int_{t^{*}}^{t}\|f(s)\| \mathrm{d} s \\
& \leq \frac{1}{a \alpha^{2}} I\left(\Phi\left(t^{*}\right)\right)+\frac{3 \varepsilon}{4}
\end{aligned}
$$

For every $t \in\left[t^{*}, T\right)$, we have

$$
\begin{aligned}
\|u(t)-\varphi\| & \leq\left\|u(t)-u\left(t^{*}\right)\right\|+\left\|u\left(t^{*}\right)-\varphi\right\| \\
& \leq \int_{t^{*}}^{T}\|\dot{u}(s)\| \mathrm{d} s+\left\|u\left(t^{*}\right)-\varphi\right\| \\
& \leq \frac{1}{a \alpha^{2}} I\left(\Phi\left(t^{*}\right)\right)+\left\|u\left(t^{*}\right)-\varphi\right\|+\frac{3 \varepsilon}{4} \\
& <\varepsilon,
\end{aligned}
$$

where the second estimation is obtained by integrating (17) on $\left[t^{*}, T\right)$. This inequality implies that $T=\infty$ and also yields $\dot{u} \in L^{1}\left(\mathbb{R}^{+}\right)$. It follows that the bounded solution $u(t)$ converges to equilibrium point $\varphi$ at infinity. 


\section{Conclusion}

In this article, we prove the convergence to equilibrium of bounded solutions to a first order gradient-like system. Our results are obtained under the affect of the low damping and perturbation terms to the asymptotic behavior of solutions at infinity.

\section{Acknowledgment}

The second author was supported by Ho Chi Minh City University of Education under grant B2017-SPS-12.

\section{References}

[1] Absil, P. A., \& Kurdyka, K. (2006). On the stable equilibrium points of gradient systems. Systems \& control letters, 55(7), 573577.

[2] Bárta, T. (2014). Convergence to equilibrium of relatively compact solutions to evolution equations. Electron. J. Differential Equations, 81, 1-9.

[3] Bárta, T., Chill, R., \& Fas̆angová, E. (2012). Every ordinary differential equation with a strict Lyapunov function is a gradient system. Monatshefte für Mathematik, 166(1), 57-72.

[4] Bégout, P., Bolte, J., \& Jendoubi, M. A. (2015). On damped second-order gradient systems. Journal of Differential Equations, 259(7), 3115-3143.

[5] Cabot, A., \& Frankel, P. (2012). Asymptotics for some semilinear hyperbolic equations with non-autonomous damping. Journal of Differential Equations, 252(1), 294322 .

[6] Chill, R. (2003). On the Lojasiewicz-Simon gradient inequality. Journal of Functional Analysis, 201(2), 572-601.

[7] Chill, R., \& Jendoubi, M. A. (2003). Convergence to steady states in asymptotically autonomous semilinear evolution equations. Nonlinear Analysis: Theory, Methods \& Applications, 53(7-8), 1017-1039.

[8] Chill, R., Haraux, A., \& Jendoubi, M. A. (2009). Applications of the LojasiewiczSimon, gradient inequality to gradient-like evolution equations. Analysis and Applications, 7(04), 351-372.

[9] Ghisi, M., Gobbino, M., \& Haraux, A. (2015). A description of all possible decay rates for solutions of some semilinear parabolic equations. Journal de Mathématiques Pures et Appliquées, 103(4), 868-899.

[10] Ghisi, M., Gobbino, M., \& Haraux, A. (2016). The remarkable effectiveness of time-dependent damping terms for second order evolution equations. SIAM Journal on Control and Optimization, 54(3), 12661294.

[11] Ghisi, M., Gobbino, M., \& Haraux, A. (2016). Finding the exact decay rate of all solutions to some second order evolution equations with dissipation. Journal of Functional Analysis, 271(9), 2359-2395.

[12] Grasselli, M., \& Pierre, M. (2012). Convergence to equilibrium of solutions of the backward Euler scheme for asymptotically autonomous second-order gradient-like systems. Commun. Pure Appl. Anal, 11(6), 2393-2416.

[13] Haraux, A., \& Jendoubi, M. A. (1998). Convergence of solutions of second-order gradient-like systems with analytic nonlinearities. journal of differential equations, 144(2), 313-320.

[14] Haraux, A., \& Jendoubi, M. A. (2007). On the convergence of global and bounded solutions of some evolution equations. Journal of Evolution Equations, 7(3), 449-470.

[15] Haraux, A., \& Jendoubi, M. A. (2015). The convergence problem for dissipative autonomous systems: classical methods and recent advances. Springer.

[16] Huang, S. Z. (2006). Gradient inequalities with applications to asymptotic behavior 
and stability of gradient-like systems (No. 126). American Mathematical Soc..

[17] Huang, S. Z., \& Takác, P. (2001). Convergence in gradient-like systems which are asymptotically autonomous and analytic. Nonlinear analysis, 5(46), 675-698.

[18] Lojasiewicz, S. (1965). Ensembles semianalytiques, I. H. E. S. Notes.

[19] Merlet, B., \& Nguyen, T. N. (2013). Convergence to equilibrium for discretizations of gradient-like flows on Riemannian manifolds. Differential and Integral Equations, 26(5/6), 571-602.

[20] Nguyen, T. N. (2013). Convergence to equilibrium for discrete gradient-like flows and An accurate method for the motion of suspended particles in a Stokes fluid (Doctoral dissertation, Ecole Polytechnique X).

[21] Simon, L. (1983). Asymptotics for a class of non-linear evolution equations, with applications to geometric problems. Annals of Mathematics, 525-571.

[22] Tran, P. M., \& Nguyen, N. T. (2017). On the convergence of bounded solutions of non homogeneous gradient-like systems. Journal of Advanced Engineering and Computation, 1(1), 61-67.

\section{About Authors}

Minh-Phuong TRAN received her Ph.D. degrees in Applied Mathematics from the University of Orléans, France, in 2012. In 2013, she joined the Department of Mathematics and Applications in Ecole Normale Supérieur , Paris, France, as a Postdoctoral Researcher. She is working at Ton Duc Thang University as a Lecturer in Faculty of Mathematics and Statistics and a member of Applied Analysis Research Group. Her current research interests include Numerical Analysis, Image Processing, Inverse Problem in Imaging, Computational Fluid Dynamics, Ordinary and Partial Differential Equations.

Thanh-Nhan NGUYEN received his Ph.D. degree in Applied Mathematics from École Polytechnique, France in 2013. He is working in Department of Mathematics, Ho Chi Minh City University of Education, Vietnam. His current research interests include Ordinary and Partial Differential Equations, Numerical Analysis and Computational Fluid Dynamics.

"This is an Open Access article distributed under the terms of the Creative Commons Attribution License, 319 which permits unrestricted use, distribution, and reproduction in any medium provided the original work is properly cited (CC BY 4.0)." 